\title{
Los caminos de la politización sexual en el campo educativo: una mirada sociosemiótica a la arena discursiva de la educación sexual en Argentina*
}

DOI: https://doi.org/10.18046/recs.i34.4221

The Paths of Sexual Politicization in the Educational Field: A Socio-Semiotic View of the Discursive Arena of Sexual Education in Argentina

Facundo Boccardi**

Universidad Nacional de Córdoba (Córdoba, Argentina)

\footnotetext{
* Este artículo ha sido elaborado a partir de la investigación en el marco de la tesis doctoral del autor defendida en el 2018, y financiada por CONICET; ha sido enriquecido en el marco del proyecto de investigación "Feminismos y pensamiento crítico. Lecturas políticas de las teorías" financiado por SECYT-UNC. Artículo de investigación recibido el 11.08.2O2O y aprobado el 12.01.2021.

${ }^{* *}$ Doctor en Semiótica por la Universidad Nacional de Córdoba (Argentina). Licenciado en Letras modernas (FFyH-UNC) (Argentina). Licenciado en Comunicación Social (FCC-UNC) (Argentina). Coordinador del Programa de Estudios de Género del Centro de Estudios Avanzados (FCS-UNC) (Argentina). Docente de Teoría Literaria (FFyH-UNC) (Argentina). Director del proyecto de investigación "Feminismos y Pensamiento Crítico. Lecturas Políticas de las Teorías" avalado y financiado por SECYT-UNC (Argentina). Correo electrónico: facundoccardi@gmail.com ORCID: https:// orcid.org/oooo-0002-8856-5203
} 


\section{Cómo citar/How to cite}

Boccardi, Facundo (2O21). Los caminos de la politización sexual en el campo educativo: una mirada sociosemiótica a la arena discursiva de la educación sexual en Argentina.

Revista CS, 34, 211-239. https://doi.org/10.18046/recs.i34.4221 


\section{Resumen}

La educación sexual en Argentina ha sido objeto de contiendas y disputas. Abordaremos desde una perspectiva sociosemiótica las relaciones entre los diferentes posicionamientos discursivos que se trabaron en los procesos de formulación de esta política pública. La sanción de la ley que obliga al sistema educativo nacional a implementar educación sexual integral en las escuelas se inscribe en un campo de disputas sedimentado históricamente donde participan formaciones discursivas relativas a la biomedicina, los derechos humanos, la religión y los movimientos feministas. El artículo indaga genealógicamente las tensiones de larga duración entre los posicionamientos feministas y los conservadurismos religiosos que atraviesan distintos campos de interlocución, disputando la legitimidad y aceptabilidad de sus argumentos. La articulación analítica de la interdiscursividad con la genealogía permite dar cuenta de los matices tácticos que desafían dicotomías y posiciones irreductibles en las luchas de poder por definir los alcances y los sentidos de la educación sexual.

\section{PALABRAS CLAVE:}

educación sexual integral, derechos sexuales, derechos (no) reproductivos, discursos, sociosemiótica

Sex education in Argentina has been the subject of contentions and disputes. From a socio-semiotic perspective, we will address the relationships between the different discursive positions that were blocked in the processes of formulation of this public policy. The sanction of the law that obliges the educational system of the entire country to implement comprehensive sex education in schools is part of a historically settled field of disputes, involving discursive formations related to biomedicine, human rights, religion, and feminist movements. The article genealogically investigates the long-term tensions between feminist positions and religious conservatisms that cross different fields of dialogue, disputing the legitimacy and acceptability of their arguments. The analytical articulation of interdiscursivity with genealogy allows us to account for the tactical nuances that challenge dichotomies and irreducible positions in the power struggles to define the scope and meaning of sexual education.

\section{KEYWORDS:}

Comprehensive Sex Education, Sexual Rights, (non) Reproductive Rights, Discourses, Socio-Semiotics 

“(...) el sexo es una categoría social impregnada de política”.

Kate Millet, La politica sexual (1970)

\section{Acerca de la sociosemiótica de las políticas de educación sexual}

Las políticas públicas que surcan el campo educativo argentino se han articulado progresivamente en la primera quincena de este siglo bajo la axiomática de la ampliación de derechos (Cfr. Feldfeber y Guy, 2011; Tedesco, 2015; Terigi, 2016). En este artículo nos proponemos indagar las operaciones discursivas que tuvieron lugar en la formulación de una política pública durante la década $2001-2010^{1}$ que condensó de manera inédita en este país el derecho a la educación con los derechos sexuales y (no) reproductivos ${ }^{2}$. Dicha política tiene como objeto la incorporación de la educación sexual integral (ESI) en todas las escuelas del sistema educativo del territorio nacional. No abordaremos en esta instancia el complejo estado actual de implementación efectiva de esta política pública, signado por la heterogeneidad y la disparidad, sino que nos dedicaremos a indagar genealógicamente sobre el sustrato discursivo de las disputas por la definición y el alcance de la ESI como una política educativa.

El posicionamiento teórico que sostiene la indagación de estas disputas de sentido acerca de la educación sexual está construido, principalmente, a través de aportes provenientes de la sociosemiótica y los estudios de género y sexualidades. Desde ese cruce específico de disciplinas, que concibe la discursividad social como un espacio privilegiado para analizar el juego de las relaciones de poder que hacen inteligible a la sexualidad (Butler, 2002; Foucault, 2007), abordaremos la sedimentación (Bajtin, 2005) de una disputa histórica por definir la educación sexual como una política pública. Consideramos que para comprender la configuración y el funcionamiento de la ESI resulta necesario reponer la densidad histórica de un espacio discursivo que

1. El punto de partida de esta indagación en términos de materialidad significante es el texto de la Ley $\mathrm{n}$. 26.150 promulgada en octubre de 2006. Pero, como veremos, la vocación diacrónica de nuestra mirada teórica y metodológica nos llevará a transitar instancias significativas del siglo XX que han dejado marcas en la memoria discursiva de la sexualidad y, a su vez, nos permitirá proyectar algunos efectos discursivos que tuvieron lugar en documentos publicados en los años subsiguientes.

2. Se utiliza la expresión "derechos sexuales (no) reproductivos" tal como fue propuesta por Josefina Brown (2008) para dar cuenta, además de los derechos sexuales, de aquellos referidos tanto a la reproducción como a la no reproducción (regulación de la propia fecundidad). 
ha sido trazado por la demarcación lábil e inestable entre los campos educativos, de la salud y las políticas demográficas, legitimando diferentes objetos de intervención, prácticas y agentes. La ubicación de la ESI en el campo educativo por parte de la ley n. ${ }^{\circ} 26.150$, sancionada en el año 2006, y la definición de los docentes como agentes legítimos para su implementación, se produce al interior de una disputa de larga duración en la que contienden distintas formaciones discursivas.

De acuerdo con la mirada teórica y metodológica que sostenemos, situada en las coordenadas de la sociosemiótica (Angenot, 1989), entendemos que los sentidos que recorreremos no se encuentran en las palabras ni en los entramados textuales, sino en las relaciones que estas superficies significantes establecen con su exterioridad. Para indagar tales relaciones recurrimos a la noción de formación discursiva (Courtine, 1981), que nos permitirá cartografiar los regímenes discursivos que han determinado lo que puede ser dicho y cómo puede ser dicho desde ciertas posiciones estabilizadas en la topología del discurso social (Angenot, 1989). Estas formaciones no son estructuras cerradas, sino que se encuentran contaminadas de manera constitutiva por elementos que provienen de otras formaciones discursivas. Para esta perspectiva analítica, la clave de la producción de sentido se encuentra en las relaciones interdiscursivas. Por esa razón, nos interesa indagar el conjunto dinámico y siempre tensionado de las formaciones discursivas, con sus relaciones de solidaridad y de enfrentamiento, que se corresponde con todo lo que puede ser dicho y pensado en una sociedad y en un horizonte epocal específicos.

Teniendo en cuenta la vocación topológica de esta perspectiva, asumimos la existencia de un campo discursivo de larga duración cuyas formaciones discursivas, tradiciones y posicionamientos han delimitado de manera recíproca y contingente la producción de sentidos acerca de la educación sexual. Al interior de ese vasto campo deslindamos un espacio analítico que conserva la heterogeneidad enunciativa de los juegos de disputa por la distribución de legitimidades y la definición de los objetos discursivos con sus densidades ideológicas específicas de aquello que, en un estado del discurso social, se considera educación sexual.

\section{La emergencia de los tópicos salud, derechos y sexualidad en las leyes argentinas}

En 1994 en Argentina tiene lugar una reforma constitucional que abre un espacio para el ingreso de la sexualidad al discurso de los derechos en consonancia con las operaciones discursivas que transcurrían en el ámbito transnacional. Así, adquieren estatus constitucional tratados, declaraciones y convenciones que dan surgimiento 
a una nueva categoría de derechos, elementos con los cuales se reconoce a los niños y adolescentes como sujetos de derechos referidos al acceso a la educación sexual. En ese espacio discursivo acerca de la sexualidad, el tema principal del debate fue el aborto y estuvo tensionado en relación con dos acontecimientos opuestos: por un lado, la incorporación a la constitución de la Convención sobre la Eliminación de Toda Forma de Discriminación Contra la Mujer (Cedaw); y por otro, el intento del entonces presidente Carlos Menem de introducir un artículo que garantizara "el derecho a la vida desde la concepción hasta la muerte natural" (Cfr. Petracci y Pecheny, 2007: 32-33).

En un marco signado por la inscripción de la sexualidad en el campo de los derechos humanos a partir de las condiciones establecidas por el discurso internacional, la propuesta de Menem introdujo la temática del aborto en la agenda política y, a su turno, dio lugar al ingreso de organizaciones feministas en la arena de debate (Cfr. Boccardi, 2020). De esta manera, los debates que precedieron la reforma constitucional se configuraron como un espacio de disputa entre dos posiciones antagónicas: feminista y católica. Con el objeto de participar en la Convención Constituyente se conformó el Movimiento de Mujeres Autoconvocadas por el Derecho a Elegir en Libertad, que reunió a 108 organizaciones feministas (Pecheny, 2011: 97). El debate implicó un despliegue discursivo de gran magnitud e instaló la temática del aborto en la agenda mediática: se publicaron solicitadas, columnas de especialistas y cartas abiertas, y se realizaron sondeos de la opinión pública (Laudano, 1998). La Convención Constituyente finalmente no incluyó ninguna cláusula que reconociera el derecho a la vida desde su concepción, ni que prohibiera el aborto (Filippini, 2011: 401).

Los debates acerca de los derechos sexuales y reproductivos (DDSSRR) formaron parte del contexto discursivo de la reforma constitucional. Si bien en el texto de la Constitución casi no hay referencias a la sexualidad en clave de derechos humanos $^{3}$, los tratados internacionales incorporados ${ }^{4}$ consagran un amplio abanico de derechos reproductivos y un conjunto más limitado de derechos sexuales. Mediante

3. La única referencia en el articulado de la Constitución Nacional de 1994 relativa a los DDSSRR aparece en el inciso 23 del artículo 75: "Dictar un régimen de seguridad social especial e integral en protección del niño en situación de desamparo, desde el embarazo hasta la finalización del periodo de enseñanza elemental, y de la madre durante el embarazo y el tiempo de lactancia" (como se citó en Monte y Gavernet, 2005: 45).

4. Estos son: la Convención sobre los derechos del niño - artículos 19, 34 y 24, inc. d y f-, la Convención sobre la Eliminación de todas las Formas de Discriminación contra la Mujer - artículos 11, 12 y 14, inc.b-, la Declaración Americana de los Derechos y Deberes del Hombre - artículo 8-, la Declaración Universal de derechos humanos - artículo 25, inc. 2-, la Convención Americana sobre derechos humanos - artículos 17 y 4, inc. 5-, el Pacto Internacional de Derechos Económicos, Sociales y Culturales - artículo 10, inc. 2-y el Pacto Internacional de Derechos Civiles y Políticos y su Protocolo Facultativo — artículo 23 y 6, inc. 5-11-. 
esta operación, el ingreso de los DDSSRR a la constitución nacional tiene lugar de manera restringida. Así, el énfasis de los tratados internacionales incorporados recae sobre los derechos reproductivos de la mujer embarazada: otorga menor desarrollo a los derechos relativos a la no reproducción, que son ubicados exclusivamente en el ámbito familiar, y limita aquellos referidos al ejercicio de la sexualidad solo a la protección de niños frente al abuso y la explotación sexual y a la trata de personas. Sin embargo, el movimiento de ingreso de los discursos internacionales de derechos humanos acerca de la sexualidad que produce esta reforma legitima un espacio discursivo en la esfera pública que permite instalar demandas de los movimientos de mujeres que permanecían postergadas (Boccardi, 2020).

En ese marco, un año después de la reforma constitucional, la Ley nacional de Salud Sexual y Procreación Responsable (SSyPR) obtiene media sanción en la cámara de diputados. Esta ley perderá estado parlamentario en 1997 y volverá a obtener media sanción nuevamente en el 2001, para finalmente lograr su sanción definitiva por la Cámara de Senadores en octubre del 2002. Durante todo ese período tuvo lugar una intensificación de los discursos acerca de la sexualidad en clave de derechos que produjo, a su vez, una "catarata de discusión y aprobación de leyes en diversas provincias y municipios del país"s (Petracci y Pecheny, 2007: 37). De acuerdo con los sondeos de la opinión pública realizados en la segunda mitad de la década de 1990, se instaló un apoyo mayoritario a la provisión de métodos anticonceptivos y la difusión de información referida a la sexualidad por parte del sistema de salud público (Petracci, 2004; Cedes, 2004). Asimismo, la sucesión de debates parlamentarios y su difusión en los medios masivos de comunicación dieron visibilidad e incrementaron el conocimiento de nociones como "DDSSRR", "derechos humanos" y "salud reproductiva", cuya articulación se volvió cada vez más recurrente. En este período la agenda mediática promovió, por primera vez, el tema de la responsabilidad del Estado en la provisión de servicios de salud pública relativos a la dimensión reproductiva de la sexualidad (Petracci y Pecheny, 2007).

2002 constituye un hito importante en la memoria de este espacio discursivo, ya que en ese año fue sancionada la Ley Nacional de SSyPR n. ${ }^{\circ} 25.673$. Este acon-

5. Las siguientes jurisdicciones sancionaron leyes con posterioridad a la reforma constitucional y previas a la Ley nacional: "Córdoba en 1996, ley que fue vetada parcialmente por el Poder Ejecutivo con el argumento de una deficiente técnica legislativa, luego fue derogada y se sancionó una nueva ley en 2003. Mendoza en 1996. Corrientes en 1996, ley muy restringida en sus objetivos y acciones, que fue ampliada en 2004 a través de una ley en la que se incorporó el suministro de anticoncepción. Chaco en 1996, ley que recibió el veto del Poder Ejecutivo y finalmente, fue aprobada con modificaciones ese año. Río Negro en 1996, norma sustituida en el año 2000 por la Ley 3450. Neuquén en 1997. Jujuy en 1999. Chubut 1999.Ciudad de Buenos Aires en 2000. La Rioja en 2000, la Ley tuvo veto parcial del Poder Ejecutivo y finalmente fue derogada en 2003. Tierra del Fuego en 2000. Santa Fe en 2001. San Luis en 2002" (Petracci y Pecheny, 2007: 106-107). 
tecimiento jurídico estuvo atravesado por un debate intenso y extenso, con fuerte protagonismo de los movimientos de mujeres y sectores de la Iglesia católica. Desde la última parte de la década de 1990, y fundamentalmente a partir del 2000, la sexualidad se configuró en un tema central de la producción discursiva de la Iglesia católica. La Comisión Episcopal Argentina (CEA) publicó una serie de documentos que sentaban un posicionamiento frente a los proyectos relativos a la salud reproductiva que circulaban en el Congreso Nacional. De esta manera, la jerarquía eclesiástica estableció un conjunto de principios que operaban como prescripciones para los proyectos legislativos:

\begin{abstract}
el matrimonio indisoluble como espacio exclusivo y la procreación como finalidad de la práctica sexual; el papel primordial e insustituible de los padres para consentir el acceso a los servicios de salud de sus hijos; la explicitación del derecho a la objeción de conciencia por parte de los prestadores de salud frente a prácticas consideradas éticamente inaceptables y la identificación "con el derecho fundamental a la vida desde la concepción y [que se excluyera] el crimen del aborto”. (como se citó en Esquivel, 2013: 87-88)
\end{abstract}

En oposición a cada uno de estos puntos, el posicionamiento de las agrupaciones de mujeres impulsaba reivindicaciones inscriptas en la tradición del feminismo de la segunda ola, centradas en su autonomía corporal a partir del control de la reproducción y el ejercicio de la sexualidad. De este modo, los colectivos de mujeres consideraban que el acceso a derechos sexuales y (no) reproductivos formaba parte del embate político contra la subordinación de las mujeres cuestionando las normas reproductivas que rigen la sociedad (Brown, 2008).

En el cruce de la contraposición histórica entre la posición feminista y la posición de la Iglesia católica, el contenido de esta ley no conforma a ninguna de ellas (Peñas-Defagó y Campana, 2011:34). La Ley es interpretada como deficiente desde los sectores vinculados al feminismo, por cuanto incorpora elementos que pueden obstaculizar el ejercicio de DDSSRR. Las principales críticas son: la mención de la "patria potestad", considerada un factor que podría coartar el acceso a información y a métodos por parte de los adolescentes menores de edad; la inclusión de la "objeción de conciencia" que exime a las instituciones privadas de carácter confesional de brindar información, prescripción o suministro de métodos anticonceptivos; el permiso que se les otorga a las escuelas privadas de impartir información referida a la sexualidad según sus valores y convicciones; y la falta de precisión en los métodos anticonceptivos que el Estado se obliga a suministrar, dado que deja abierta la posibilidad de que ciertos métodos considerados "falsamente abortivos" no sean suministrados (Lipszyc, citada en Peñas-Defagó y Campana, 2011: 38-39). Del 
lado de los sectores religiosos, la CEA publicó un documento en el cual se detallan aquellos aspectos que se oponen a las mencionadas prescripciones centradas en la reproducción matrimonial. Ellos son la intencionalidad "escondida" de legalizar el aborto; la disposición de "métodos anticonceptivos no conformes al orden natural"; la violación de "de la patria potestad y el derecho natural de los padres a la educación de los hijos"; y la exclusión de "la educación por la castidad" prematrimonial (como se citó en Peñas-Defagó y Campana, 2011:38-39). Las tensiones entre ambas posiciones se inscriben en una larga tradición de oposición y, a su vez, anticipan fundamentalmente el posicionamiento de la Iglesia católica frente a la Ley de ESI.

Por otro lado, la defensa de la Ley n. ${ }^{\circ} 25.673$ en el contexto de su sanción se sostuvo principalmente con argumentos sanitaristas desde el Ministerio de Salud de la Nación. Durante el período de debate parlamentario, Ginés González García, por entonces titular de esa cartera, había instalado con sus declaraciones ante la prensa la necesidad urgente de una política nacional que abordara la salud sexual y reproductiva para reducir la mortalidad causada por el aborto y la transmisión de infecciones sexuales (Boccardi, 2010).

El poder legislativo nacional se constituyó en ese período histórico en un espacio de disputa clave en la producción de legitimidad acerca de las intervenciones estatales en el campo de la sexualidad. Aunque lleva en su título el término "salud sexual", esta ley se inscribe claramente en la tradición de la "salud reproductiva", cuyo objeto es desarrollar estrategias de regulación de los comportamientos sexuales individuales que apuntan a la planificación responsable de la reproducción y a la prevención frente a la transmisión de infecciones. Los elementos léxicos presentes en la Ley y su decreto de reglamentación (Decreto 1282/2003) dan cuenta de la filiación a esa tradición discursiva. De ese modo, en la fundamentación de los objetivos se menciona "el derecho a la planificación familiar", definido por la Organización Mundial de la Salud (OMS) como un modo de vida que promueve "la salud y el bienestar de la familia" (Decreto 1282/2003). De la misma forma, estos documentos incluyen los términos "comportamiento reproductivo" y "salud reproductiva"; este último aparece definido, en línea con el planteamiento de la OMS, como "un estado general de bienestar físico, mental y social" (Decreto 1282/2003). En cambio, la noción de "salud sexual" no es mencionada ni desarrollada en estos textos, ni se mencionan los términos asociados de forma recurrente a ella como "bienestar sexual" o "placer sexual". Con un sentido claramente centralizado en la dimensión reproductiva, las estrategias de intervención consisten, por un lado, en la consejería y transmisión de información relativa a la reproducción y a la transmisión de infecciones; y por otro, en la prescripción, el suministro y el seguimiento de elementos y métodos anticonceptivos. 
Como lo hemos visto, los puntos relevantes de conflicto de la sanción de esta ley han sido las nociones de "patria potestad" y "objeción de conciencia", así como la posibilidad de las instituciones educativas de gestión privada de cumplir con la norma "en el marco de sus convicciones" (Ley 25.673, 2002). Estos aspectos anticipan los conflictos que tendrán lugar en torno a la sanción de la Ley de ESI años más tarde, ya que ponen en juego la tensión entre la concepción de los niños como sujetos de derecho y el rol de las familias y otras instituciones dedicadas a su cuidado.

Con respecto a las familias, la Ley 25.673 cita a la Convención sobre los derechos del niño $(\mathrm{CDN})^{6}$ en un planteo que concilia el respeto a la institución de la "patria potestad" con una concepción de los niños como sujetos de derecho. El artículo $4 .{ }^{\circ}$ de esta Ley ${ }^{7}$ constituye una respuesta interdiscursiva al discurso católico que atribuía a la norma el avance sobre los derechos de los padres a regular el acceso a información acerca de la sexualidad por parte de sus hijos. Las interpretaciones de especialistas inscriptos en el campo de la educación y del derecho sostienen que este artículo no niega la "patria potestad", sino que resignifica las responsabilidades que la definen al enmarcarla en el ejercicio de derechos a la información, a la educación y a la salud por parte de los niños tal como lo establece la CDN (Cfr. Faur, 2007: 54-55; Minyersky, 2003). Sin embargo, la Ley 23.849, citada en el artículo que aprueba y ratifica la CDN en el marco jurídico nacional, plantea entre sus "reservas y declaraciones" que "las cuestiones vinculadas con la planificación familiar atañen a los padres de manera indelegable de acuerdo a [sic] principios éticos y morales" (Ley 23.849, 1990). Las "reservas" expresadas por esta ley de 1990 se refieren a la única mención a lo largo de todo el articulado de la CDN referido a la transmisión de la información sobre sexualidad a los niños ${ }^{8}$. Esta referencia pone de manifiesto la presencia de un nudo problemático en el discurso jurídico que condensaba la oposición de la Iglesia católica ante las posibilidades de avance de la educación sexual en las instituciones públicas, legitimadas por los discursos internacionales de derechos humanos.

6. Esta convención fue adoptada en 1989 por la Asamblea General de las Naciones Unidas en Nueva York y ratificada por Constitución Nacional en la reforma de 1994.

7. Artículo $4 .^{\circ}$. La presente ley se inscribe en el marco del ejercicio de los derechos y obligaciones que hacen a la patria potestad. En todos los casos se considerará primordial la satisfacción del interés superior del niño en el pleno goce de sus derechos y garantías consagrados en la Convención Internacional de los derechos del niño (Ley 23.849).

8. La CDN se refiere a la sexualidad de los niños solo en dos situaciones: cuando menciona, como hemos visto, el derecho a la "planificación familiar" en el artículo 24; y cuando se refiere al derecho expresado en términos negativos a no ser objeto de abuso o explotación sexuales (CDN, 1989). 
Otro de los aspectos que opera de manera restrictiva es la posibilidad que atribuye la Ley tanto a profesionales e instituciones de adecuar el cumplimiento de la norma según su "conciencia" o sus "convicciones". Los artículos 9. y 10. de la Ley establecen excepciones destinadas a las instituciones confesionales: en el caso de las instituciones de salud se las exime de prescribir y suministrar elementos y métodos anticonceptivos; mientras que a las instituciones educativas se les permite adecuar la transmisión de información sobre sexualidad al "marco de sus convicciones" (Ley 25.673). El decreto reglamentario, por su parte, introduce la noción de objeción de conciencia que individualiza la excepción del cumplimiento con las disposiciones (Decreto 1282/2003). Esta individualización de la excepción resulta significativa ya que, en lugar de eximir a las instituciones privadas de carácter confesional de cumplir con lo establecido por la norma, limita el alcance de la excepción a los individuos que se declaren "objetores de conciencia", aunque lo expande a las instituciones de gestión pública. Por último, si bien el alcance de la figura de la objeción de conciencia se limita a los profesionales de la salud ${ }^{9}$, la particularización de la relación con la norma según las convicciones de los miembros de la institución se replicó en la Ley de ESI instalándose como uno de los elementos de debate.

\section{El territorio de disputas de la ESI: tensiones y avances}

La sanción de la Ley de SSyPR y la puesta en funcionamiento del programa homónimo durante el 2003 aparecen como los signos de un desplazamiento en el posicionamiento del Estado argentino con respecto al abordaje de la sexualidad. Entre los indicios de ese desplazamiento aparece el posicionamiento público del ministro de salud, ratificado en su cargo con el inicio de la nueva gestión del gobierno nacional ${ }^{10}$. Este ministro enfatiza públicamente la necesidad urgente de implementar políticas públicas de alcance nacional para garantizar el acceso a los derechos sexuales y (no) reproductivos de la población (Cfr. Brown, 2014).

La puesta en marcha de este programa constituye el antecedente más significativo de las iniciativas que impulsarán normativas específicas en educación sexual. Además de este impulso en el marco de las políticas públicas, la educación sexual

9. La objeción de conciencia es incorporada en otras leyes referidas a la salud reproductiva tales como la Ley nacional de Anticoncepción Quirúrgica (26.130) sancionada en 2006.

10. Ginés González García había asumido al frente de la cartera nacional de salud con la presidencia transitoria de Eduardo Duhalde, y fue ratificado el 25 de mayo de 2003 junto con la asunción de la presidencia de Néstor Kirchner. 
se volvió a instalar con mucha fuerza como tema de debate en la prensa nacional ${ }^{11}$ (Boccardi, 2010). Al mismo tiempo, en algunos sistemas educativos jurisdiccionales se implementaron acciones de educación sexual destinadas principalmente a brindar herramientas a los estudiantes de cuidado de la salud sexual y reproductiva (Cfr. Wainerman, Virgilio y Chami, 2008).

Durante este período previo a la sanción de la Ley nacional de ESI se configura un espacio de disputas donde se cruzan principalmente discursos provenientes del Estado, la Iglesia católica y los movimientos feministas. Los imperativos relacionados con la salud reproductiva y la trasmisión de VIH-SIDA instalados desde la década de 1990 en la discursividad social aparecen con mucha fuerza. El llamado "embarazo adolescente" adquiere un lugar primordial en la prensa nacional, que le atribuye de manera recurrente la condición de epidemia y posiciona la educación sexual escolar como una estrategia para combatirla (Boccardi, 2010).

Por el lado de los movimientos de mujeres y feministas, los derechos sexuales y (no) reproductivos emergen como la demanda principal realizada en los encuentros nacionales de mujeres ${ }^{12}$ desde el año 2003 (Alma y Lorenzo, 2009). Como consecuencia de esos encuentros, en mayo del 2005 se organizó la Campaña Nacional por el Derecho al Aborto, cuyo lema fue "educación sexual para decidir, anticonceptivos para no abortar, aborto legal para no morir" (Bellucci, 2014: 390). Este lema histórico, que le exige al Estado la garantización de derechos (no) reproductivos para las mujeres, posiciona la educación sexual en un lugar estratégico cardinal. El posicionamiento de esta demanda de intervención estatal en educación sexual se encuentra legitimado tanto por la relevancia de la salud reproductiva de las mujeres, atribuida por el discurso internacional de derechos humanos, como por la función preventiva que le otorga la Ley de SSyPR (Boccardi, 202O). En esta coyuntura específica, las demandas de estos sectores del feminismo y los movimientos de mujeres acentuaban las consecuencias negativas de la sexualidad centralizadas en la mujer, y concebían

11. En el 2004, la prensa difundió los resultados de una encuesta de alcance nacional realizada por el Instituto Social y Político de la Mujer (ISPM) y la United Nations Funds for Population (UNFPA): 96, 9\% de encuestados estuvieron a favor de incluir la educación sexual en las escuelas.

12. Se trata de encuentros autoorganizados que se realizan anualmente en Argentina desde 1986, cada vez más masivos y significativos para la agenda política de los feminismos en Latinoamérica: "La modalidad del Encuentro Nacional de Mujeres es única en el mundo, y eso permite que cada año nos sumemos de a miles: es autoconvocado, horizontal, federal, autofinanciado, plural y profundamente democrático. Cada año, al encontrarnos intercambiamos nuestras vidas, nuestras experiencias y convertimos problemas que parecen individuales en un problema de todas. Eso nos ayuda a encontrar los caminos para resolver nuestros sufrimientos. En el encuentro también expresamos nuestras luchas, la que damos en la fábrica, la casa, el barrio, el campo, la escuela, la facultad, la ciudad, etc." (Extraído de https://razonyrevolucion. org/sin-brujula-cronica-del-32-encuentro-nacional-de-mujeres-chaco-2017/) 
la educación sexual como una herramienta para su prevención. De acuerdo con esta concepción negativa de la sexualidad, la posición feminista respecto de la educación encuentra un lugar de convergencia con la posición sanitarista del Estado, dado por la presencia de un axioma que articula la siguiente premisa: la educación sexual previene los problemas de la sexualidad.

Por el lado de la Iglesia católica, se multiplicaron las intervenciones mediante la prensa y la publicación de documentos con el objeto de incidir en las propuestas de educación sexual que circulaban en los espacios legislativos. La legislatura de la Ciudad Autónoma de Buenos Aires fue un escenario con gran repercusión nacional entre 2004 y 2006, ya que fueron discutidas diferentes iniciativas de educación sexual. Desde los sectores del catolicismo, y en coherencia con las objeciones a la Ley de SSyPR, se planteaba que la educación sexual propuesta implicaba una intromisión y un avasallamiento por parte del Estado en el ámbito privado de las familias (Boccardi; Boccardi, 2011). Así, la Iglesia católica sostenía que la prescripción de contenidos obligatorios sobre sexualidad en todas las escuelas violaba la patria potestad de los padres y la libertad de enseñanza de los colegios confesionales (Esquivel, 2013:49) ${ }^{13}$. Con respecto a los contenidos de educación sexual, sostenían que estos tendían a subvertir el orden biológico natural, producir confusión de identidades, reducir la sexualidad al coito e impulsar a la juventud a la promiscuidad y la inmoralidad. En contra de los argumentos sanitaristas esgrimidos desde el feminismo y los agentes del Ministerio de Salud, sostenían que la implementación masiva de la educación sexual en las escuelas "aumentaría los índices de contacto sexual y, en consecuencia, de embarazos no deseados y enfermedades sexuales" (como se citó en Esquivel, 2013: 49). De esta manera, la posición católica comparte la concepción negativa de la sexualidad, entendida como una problemática social presupuesta por el feminismo y el Estado, pero se articula en una premisa que invierte los efectos atribuidos a la educación sexual al considerarla promotora de la actividad sexual y del incremento de sus consecuencias problemáticas.

La literatura especializada plantea que durante este período de gobierno nacional se debilitó la capacidad de la Iglesia católica para influir en las políticas, dado que el gobierno mantuvo una actitud prescindente y distante con la jerarquía eclesiástica (Pecheny, Jones y Ariza, 2016: 73; Malimacci, 2012: 186). En la historia argentina, las tensiones entre los actores estatales y eclesiásticos en torno a la sexualidad emergieron con la discusión legislativa y posterior sanción de la Ley de divorcio durante

13. Además de la "objeción de conciencia" que hemos mencionado más arriba, la patria potestad y la defensa del ejercicio de la libertad religiosa componen una tríada argumental que se repite a lo largo de toda la región en las voces desde la Iglesia católica para poner en cuestión la vigencia de DDSSRR (Puga y Vaggione, 2013). 
el gobierno nacional de Raúl Alfonsín, en los años 1986 y 1987 (Pecheny, 2010; Esquivel, 2004). Esta situación se extendió con diferentes intensidades y abarcó tópicos relacionados con la anticoncepción, la educación sexual y el matrimonio entre personas del mismo sexo. Durante estas décadas, el activismo religioso ha seguido una estrategia de "politización reactiva" (Vaggione, 2005; 2011) que consiste en posicionamientos adversativos frente a las demandas y los avances de los movimientos que luchan por el acceso a derechos sexuales y (no) reproductivos.

En el campo de la educación, las posibilidades de incidencia de la Iglesia católica sufrieron variaciones significativas con el gobierno nacional que inició en 2003. Durante la década de 1990, la injerencia de la Iglesia católica había sido muy profunda. Dos acontecimientos sirven para poner de relieve esta situación: en primer lugar, la condensación en la Ley Federal de Educación de muchas demandas históricas de la Iglesia - tales como la inclusión de la familia como principal y primera educadora; la inclusión de la dimensión religiosa en los objetivos de formación; el financiamiento estatal de los sueldos docentes de las escuelas de gestión privada; y la inclusión de los establecimientos educativos privados como parte de la educación pública- (como se citó en Torres, 2014: 80 $)^{14}$; y en segundo lugar, el éxito que tuvieron las presiones de la Iglesia para modificar el contenido de $\operatorname{los} \mathrm{CBC}^{15}$, adecuándolos a la transmisión de su ideario. En el período abierto con la gestión nacional de Kirchner, las posibilidades de presión de la Iglesia fueron más débiles, aunque en el espacio específico de la educación sexual continuaron siendo significativas. El debate parlamentario de la Ley nacional de ESI se dispuso de manera semejante al que tuvo lugar en la legislatura porteña: actores provenientes de la jerarquía eclesiástica y de ONG afines intervinieron de formas activa y articulada con el objeto inicial de impedir la aprobación de los proyectos presentados. Entre marzo del 2005 y mayo del 2006, fueron presentados ocho proyectos parte de diputados pertenecientes a diferentes sectores partidarios. Luis Zamora, de Autodeterminación y Libertad, presentó el primer proyecto el 7 de marzo de 2005. Pasado cerca de un mes, se presentó un proyecto que reunió a diputados de partidos de izquierda - Izquierda Unida (IU), Partido Socialista (PS) y Proyecto Social Alternativo (PSA)-, de la Unión Cívica Radical (UCR) y del partido Argentinos por una República de Iguales (ARI). En el

14. Con respecto a las familias, la Ley sostiene que "las acciones educativas son responsabilidad de la familia, como agente natural y primario de la educación”; y acerca de la inclusión de la religión como objetivo educativo plantea que "El Sistema Educativo posibilitará la formación integral y permanente del hombre y la mujer, (...) que se realicen como personas en las dimensiones cultural, social, estética, ética y religiosa" (Ley 24.195, 1993).

15. Los contenidos básicos comunes (CBC) son un marco curricular unificado para la Educación General Básica (EGB). 
2006, ingresaron seis proyectos más: uno firmado por Marta Maffei, del ARI; otro, por Juliana Marino del Frente para la Victoria (FPV); otro, por María del Carmen Rico del Partido Justicialista (PJ); otro, presentado por representantes de Propuesta Republicana (PRO) junto a Guardia Peronista; y dos más presentados por representantes de Fuerza Republicana.

A excepción del proyecto presentado por Luis Zamora, que fue girado a cinco comisiones, los proyectos solo fueron tratados en dos: Educación, en un primer momento; y luego Familia, Mujer, Niñez y Adolescencia. Si bien las iniciativas contenían perspectivas heterogéneas acerca del alcance de la educación sexual, los borradores de la comisión de Educación contemplaron la incorporación obligatoria de la ESI en todas escuelas de gestión pública y privada desde nivel inicial hasta el secundario. El proceso fue expeditivo y no se produjeron tensiones significativas en esta comisión, pero no ocurrió lo mismo en la de Familia, Mujer, Niñez y Adolescencia: durante el tratamiento en esa comisión, la Iglesia católica presionó - mediante la irrupción en las reuniones de la comisión - por la distribución de panfletos opuestos a la educación sexual (Cfr. "Sesión interruptus", 2005) y la publicación de un documento de la CEA que instaba a los legisladores a "elaborar proyectos e iniciativas concretas inspirados en el Evangelio" y a no promulgar leyes que promovieran "costumbres o antivalores [contrarios a] la dignidad de la persona" (como se citó en Esquivel, 2016:134-135). Además, el documento establecía que la familia constituye el espacio por excelencia para la educación sexual de los hijos, mientras que el Estado despeña un rol subsidiario ${ }^{16}$.

Tras varias semanas de intenso debate, las legisladoras del ARI, el FPV y el socialismo aprobaron un proyecto que rechaza la inclusión de la objeción de conciencia y el ejercicio de la patria potestad exigidas por la Iglesia, mientras conservaba las especificaciones incluidas inicialmente, esto es, la obligatoriedad en todo el sistema educativo desde nivel inicial. Ante ello, la diputada del Interbloque Provincias Unidas de Buenos Aires, Nélida Morales, promovió un dictamen de minoría que elevaba la edad mínima para recibir educación sexual a 14 años y garantizaba la posibilidad de exención de estudiantes según las convicciones de su familia (como se citó en Esquivel y Alonso, 2015).

Como parte de este escenario de disputa, en los primeros meses del 2006 la Comisión de Educación de la CEA publicó el documento "El desafío de educar en el amor", en el cual se sistematizaba el posicionamiento de la jerarquía eclesiástica 
frente a la educación sexual. Allí se insistía con las nociones de patria potestad, principio de subsidiariedad, libertad de enseñanza y autonomía de los colegios religiosos. También avanzaba en el plano de los contenidos de la educación sexual en tanto establecía que "el amor y la fecundidad" conforman las bases constitutivas de la sexualidad; que la "castidad" y el "método natural" son las mejores herramientas para controlar la natalidad; y que la "vida sexual" debe restringirse al matrimonio (como se citó en Esquivel, 2013: 72).

A pesar de estas presiones sistemáticas, la mayoría de las expresiones partidarias (FPV, ARI, UCR, PS) apoyaron en el debate parlamentario el proyecto que desoía las prescripciones de la Iglesia. En esta instancia, las únicas objeciones provinieron de los diputados Esteban Bullrich, del PRO, y Eusebia Jerez y Roberto Lix Klett, ambos de Fuerza Republicana. De ellos, solo Lix Klett mantuvo una posición activa en el debate, mientras que las intervenciones de Bullrich y Jerez fueron poco relevantes $\mathrm{y}$ ambos terminaron por votar de manera positiva ${ }^{17}$.

Las intervenciones más significativas en el debate del 16 de agosto de 2006, que terminó con la media sanción a la Ley de ESI, fueron la de Marta Maffei, diputada del ARI por Buenos Aires, y la de Roberto Lix Klett, diputado por Tucumán y titular del único voto negativo a la media sanción de la Ley ${ }^{18}$. Resulta interesante la comparación de ambas intervenciones ya que, si bien se ubican en posicionamientos opuestos, comparten una concepción de la sexualidad (Cfr. Dvoskin, 2013). En el caso de Lix Klett, se despliega una crítica a los efectos negativos que producen las prácticas sexuales. Así, desde una fuerte filiación discursiva con el enfoque de riesgo de la biomedicina, la sexualidad es entendida como un problema de salud que amenaza la sociedad, y la educación sexual escolar es un potenciador de dicha amenaza porque "acelera el tiempo del comienzo sexual" (Dvoskin, 2013: 86). En el caso de Maffei, la educación sexual es presentada como un "derecho" de los jóvenes y la noción de sexualidad es mencionada como un "fenómeno integral que involucra aspectos biológicos, psíquicos, sociales y afectivos" (como se citó en Dvoskin, 2013: 89). Sin embargo, junto con esta retórica de derechos son recurrentes en la intervención de la diputada términos portadores de rasgos negativos tales como "padecer",

17. Retomamos a continuación aportes del análisis de los discursos de los diputados Maffei y Lix Klett realizados por Gabriel Dvoskin (2013).

18. Intervinieron once diputados, además de Maffei y Lix Klett: Blanca Inés Osuna, diputada por Entre Ríos; Lucía Garín de Tula, diputada por Catamarca; María del Carmen Rico, diputada por Buenos Aires; María Angélica Torrontegui, diputada por San Luis; Eusebia Antonia Jerez, diputada por Tucumán; Esteban José Bullrich, diputado por la Capital; Silvia Augsburger, diputada por Santa Fe; Alicia Marcela Comelli, diputada por Neuquén; y Marcela Virginia Rodríguez, diputada por la Capital. 
"enfermedades", “violencia”, "trastornos", "víctima”, "victimario” y "abuso", que presentan la sexualidad como un tema problemático (Dvoskin, 2013: 87).

A pesar de las marcadas diferencias que emplazan a ambos enunciadores en posiciones diametralmente opuestas con respecto a la educación sexual, ambos discursos sostienen una noción de sexualidad asociada a los problemas derivados de las consecuencias negativas de las prácticas sexuales; esta coincide con la perspectiva sanitarista instalada en el discurso mediático que difundía los aumentos de las tasas de embarazo adolescente y los índices de infecciones de trasmisión sexual (Boccardi, 2013). Sin embargo, el texto de la Ley que obtendría media sanción en el marco de ese debate produciría un desplazamiento desde la tradicional perspectiva sanitarista, centrada en la concepción de la sexualidad como un problema demográfico, hacia una enfocada en el derecho de los individuos. La pregnancia discursiva de ese desplazamiento, posibilitada por un abanico de leguajes disponibles que hemos recorrido, incluiría otras disputas de sentidos en el campo educativo.

A partir de la media sanción en la cámara baja, el derrotero de la Ley fue más simple. En octubre de 2006, la ESI adquirió estatus legal casi por unanimidad en la cámara alta. Esta vez, el único voto opositor fue de la senadora de San Luis Liliana Negre de Alonso, quien justificó su negativa repitiendo el argumento católico de la subsidiariedad del Estado: "El proyecto margina a las familias a las que relega a un rol secundario" (intervención en el Senado de la Nación, 4 de octubre de 2006). La sanción de la Ley obtuvo una respuesta articulada desde diferentes sectores de la jerarquía eclesiástica, que volvieron a traer al terreno de disputa los argumentos que venían esgrimiendo en contra de la intervención del Estado en la educación relativa a la sexualidad de los estudiantes. En ese sentido, un grupo de obispos apeló a la inconstitucionalidad de esta normativa alegando que se estaba legalizando una "intromisión autoritaria del Estado" en un espacio propio de los padres "por derecho natural” ("Si es sexual, nada de educación", 2006). Asimismo, otras voces individuales, pertenecientes a la jerarquía de esta institución, calificaron a la propuesta como reduccionista dado que, sostenían, omitía valores trascendentes y anticiparon que incidiría de forma negativa en tanto promovería la actividad sexual de la juventud e incrementaría las enfermedades y los embarazos.

\section{Viejas disputas y nuevas articulaciones: la Ley de ESI}

El texto de la Ley nacional n. ${ }^{\circ} 26.150$ se encuentra atravesado por estas tensiones con la Iglesia católica, al tiempo que produce articulaciones con el discurso de los derechos humanos - y de modo puntual, con los DDSSRR - que resultaban inéditas 
en el campo de la educación. La sanción de esta ley se inscribe en un marco normativo específico que incluye, además de la mencionada Ley de SSyPR, las contemporáneas Ley Nacional de Educación ( . $^{\circ}$ 26.206) sancionada también en el 2006 y la Ley de Protección Integral de los Derechos de Niños, Niñas y Adolescentes (n. ${ }^{\circ}$ 26.061), sancionada un año antes. Este marco normativo se vale del lenguaje de los derechos humanos dotado de aceptabilidad y legitimidad en ese contexto político (Cfr. Barros, 2012) para instalar la definición de los niños y adolescentes como sujetos de derecho. Esta operación que había sido formulada por la CDN en 1989, aprobada por la República Argentina y luego ratificada en la reforma constitucional de 1994, se concretiza en el marco normativo nacional recién con la Ley n. ${ }^{\circ} 26.061$, que instala el reconocimiento formal de los niños y adolescentes como sujetos plenos de derecho (Llobet, 2010). En coherencia con ello, la reestructuración normativa del sistema educativo a partir de la derogación de la Ley Federal de Educación (n. ${ }^{\circ}$ 24.195/93) y la sanción de la Ley Nacional de Educación define la escuela no solo como un espacio de adquisición de conocimientos, sino también de construcción de ciudadanía. La perspectiva de derechos que atraviesa esta ley incorpora la sexualidad como un componente de los derechos humanos y establece, entre los fines y objetivos de la política educativa nacional, el "Brindar conocimientos y promover valores que fortalezcan la formación integral de una sexualidad responsable" (Ley n. $\left.{ }^{\circ} 26.206,2006\right)$.

La operación más significativa que se produce en la Ley $n .^{\circ} 26.150$ consiste en la institución de la ESI como un derecho de los “educandos”. Con ello, convergen por primera vez en la historia nacional del sistema educativo el reconocimiento de los derechos sexuales y reproductivos como derechos humanos, y el de los educandos como sujetos de tales derechos: "Artículo 10.- Todos los educandos tienen derecho a recibir educación sexual integral [...]" (Ley n. $\left.{ }^{\circ} 26.150,2006\right)$.

Durante el debate de la Ley, la preeminencia en la agenda mediática del discurso sanitarista, que enfatizaba la necesidad de la educación sexual como una herramienta para paliar los problemas asociados a la sexualidad de los adolescentes y jóvenes frente a las posiciones reactivas de la Iglesia, había desplazado a un plano de poca visibilidad las posiciones de las organizaciones feministas y otros movimientos sociosexuales que afirmaban una perspectiva de derechos. Finalmente, esta ley define como sujetos de derecho a la ESI a todos los estudiantes del sistema educativo formal de cualquier jurisdicción y de cualquier tipo de gestión. Esta definición del sujeto receptor de ESI, legitimada por el discurso de los derechos humanos, limitó definitivamente, y por primera vez en las legislaciones vernáculas, el alcance atribuido a la patria potestad para vigilar y determinar los contenidos sobre sexualidad que reciban los estudiantes. 
Por otra parte, la Ley de ESI también incluye un artículo que disipa este movimiento a favor de los DDSSRR de los estudiantes con una concesión dotada de cierta ambigüedad, que responde a las presiones ejercidas por la Iglesia católica.

Artículo $5^{\circ}$. [...] Cada comunidad educativa incluirá, en el proceso de elaboración de su proyecto institucional, la adaptación de las propuestas a su realidad sociocultural, en el marco del respeto a su ideario institucional y a las convicciones de sus miembros. (Ley n. $\left.{ }^{\circ} 26.150,2006\right)$

Al igual que con lo ocurrido en la Ley de Salud Sexual, este artículo habilita la posibilidad de "adaptación" de las propuestas emanadas desde Programa Nacional de ESI según el "ideario institucional" y las "convicciones de sus miembros". De manera específica, en esta ley la posibilidad de adaptación se extiende a "cada comunidad educativa", mientras que en la precedente se refería exclusivamente a las instituciones "privadas" y "confesionales". Sin embargo, la coincidencia léxica entre ambas leyes, junto con la recurrencia del sintagma "ideario institucional" en los documentos de las instituciones confesionales, limita claramente a esos actores la destinación de dicha posibilidad de "adaptación". Los alcances y límites de la "adaptación" constituirían un objeto de disputa en el proceso de implementación de la ESI que continua vigente en nuestros días.

Además de estos aspectos significativos, se trata en términos generales de un articulado breve que plantea directrices generales, pero no avanza de forma significativa en la explicitación de los contenidos a ser impartidos ni en el diseño metodológico de su implementación. Con respecto a ambos puntos, la Ley establece algunos indicios que funcionarían como orientadores de la elaboración de la propuesta educativa, mediados por otras instancias de disputa. La definición de la ESI, las referencias a un conjunto normativo nacional e internacional, la formulación de los objetivos del Programa Nacional de ESI, las indicaciones generales para la implementación en cada jurisdicción y la prescripción de espacios de formación para "los padres" permiten trazar las coordenadas discursivas del posicionamiento de la Ley en la trama de más amplitud que hemos recorrido.

El artículo 1. incluye en su última oración la definición de ESI, además de definir el sujeto beneficiario de esta política pública: "A los efectos de esta ley, entiéndase como educación sexual integral la que articula aspectos biológicos, psicológicos, sociales, afectivos y éticos" (Ley n. $\left.{ }^{\circ} 26.150,2006\right)$. Se trata de la única definición de ESI que aparece en esta normativa y operaría como el punto de partida para el desarrollo conceptual que tendría lugar en los sucesivos documentos. La definición retoma el criterio de articulación entre diferentes aspectos de las definiciones de 
sexualidad producidas en el marco de la OMS; tanto en aquel como en este caso, la integralidad es definida a partir de la adición de elementos o dimensiones que establecen relaciones interactivas entre sí: "La sexualidad es el resultado de la interacción de factores biológicos, psicológicos, socioeconómicos, culturales, éticos y religiosos o espirituales" (OPS y OMS, 2000: 8).

Las similitudes entre ambos listados de elementos dan cuenta de un posicionamiento que remite a la formación discursiva de los derechos humanos, y se presenta como superador de las visiones consideradas reduccionistas por limitar la sexualidad a su dimensión bioanatómica y abordarla desde la prevención de riesgos. Resulta significativa, en el caso de la definición de ESI, la inclusión de aspectos "afectivos" que constituirán un componente relevante en los contenidos curriculares propuestos a posteriori y la exclusión de los factores "religiosos o espirituales". Con respecto a este último punto, la exclusión se inscribe en la relación que establece la normativa con el posicionamiento de la Iglesia católica. La ausencia - o la poca jerarquía-del componente religioso en el concepto de sexualidad que sostiene esta política pública constituyó uno de los reclamos constantes emitidos desde la Iglesia que la llevarían a impugnar la condición de integral esgrimida desde la Ley. En ese sentido, la ausencia de lo religioso esta normativa y en la Ley nacional de educación es leída desde la jerarquía eclesiástica como una omisión que reduce la perspectiva alcanzada en las normativas precedentes ya derogadas. ${ }^{19}$

Con respecto a los objetivos del Programa Nacional de ESI creado por esta ley, se encuentran enunciados en el artículo $3^{\circ}$.

a. Incorporar la educación sexual integral dentro de las propuestas educativas orientadas a la formación armónica, equilibrada y permanente de las personas;

b. Asegurar la transmisión de conocimientos pertinentes, precisos, confiables y actualizados sobre los distintos aspectos involucrados en la educación sexual integral;

c. Promover actitudes responsables ante la sexualidad;

d. Prevenir los problemas relacionados con la salud en general y la salud sexual y reproductiva en particular;

e. Procurar igualdad de trato y oportunidades para varones y mujeres. (Ley n. ${ }^{\circ} 26.150$, 2006)

19. En febrero de 2007, la Comisión Episcopal de Educación Católica (CEEC) publicó un documento titulado "La Iglesia y la nueva Ley de Educación Nacional": allí se menciona que la nueva ley de educación propone como objetivo la "formación integral de la persona en todas sus dimensiones", pero no se incorpora de forma explícita la "dimensión religiosa"; mientras que la Ley Federal de Educación (n. ${ }^{\circ}$ 24.195/93) "sí la reconocía en forma explícita" (CEEC, 2007). 
Estos objetivos permiten establecer relaciones discursivas con el campo de disputas que tuvo lugar en el proceso de debate y sanción de la Ley, y con un entramado histórico de discursos acerca de la educación sexual, la salud sexual y reproductiva, y los derechos. Por un lado, la "transmisión de conocimientos" aparece como el principal rasgo definitorio de la educación sexual en su tradición histórica. Como lo hemos visto, en la historia argentina de este campo se han disputado el alcance y la inscripción disciplinar de esos conocimientos, y se ha prescripto en varias oportunidades que la educación sexual no debe limitarse solo a su transmisión, sino que ha de incorporar componentes del plano moral y afectivo; empero, en ninguna propuesta se ha desplazado a este rasgo de su función estructurante. En este caso, el tema de los conocimientos no se encuentra enunciado, pero los adjetivos que lo acompañan operan como criterios de cientificidad que permiten inscribirlos en la tradición de la biomedicina que atraviesa los discursos acerca de la salud sexual y reproductiva. En coherencia con ello, la prevención de los problemas relacionados con la salud sexual y reproductiva, expresada en el objetivo "d", ha constituido históricamente el propósito central de las intervenciones desde perspectivas sanitaristas. Asimismo, el sintagma "salud sexual y reproductiva" constituye, como lo vimos arriba, una de las marcas de un posicionamiento discursivo que privilegia la dimensión reproductiva de la sexualidad y codifica las intervenciones en clave de prevención de riesgos. Por su parte, el objetivo "c" enuncia la promoción de "actitudes responsables ante la sexualidad" retomando la noción de responsabilidad individual propuesta por el marco conceptual de la "promoción de la salud" formulado por la OMS, que rige su discurso sobre salud sexual. Esta articulación con el posicionamiento discursivo que despliega la promoción de la salud sexual hace posible una ampliación de la concepción de la sexualidad restringida a lo reproductivo. Finalmente, el último objetivo de este artículo incorpora "la igualdad de trato y oportunidades para varones y mujeres". Este enunciado remite al discurso de los derechos de las mujeres que ha incorporado la reivindicación igualitarista en las instancias internacionales referidas a los derechos humanos desde la última porción del siglo pasado. La importancia de este objetivo radica en que es el único que incorpora un enfoque de derecho, de tal modo que hace posible un descentramiento de la noción biomédica de salud que aparece como predominante, y además incorpora un enfoque de género que había constituido uno de los aspectos más resistidos en las tensiones con la Iglesia católica. Si bien el término "género" está ausente en esta ley como efecto de esas tensiones ${ }^{20}$, este objetivo, junto con el marco normativo internacional incluido en el 
artículo 2. ${ }^{\circ}$, permitirían la inclusión de una perspectiva de género en los Lineamientos Curriculares para la Educación Sexual Integral (2008).

Con respecto al marco normativo, el artículo $2 .^{\circ}$ sitúa en el espacio educativo un conjunto de normas nacionales e internacionales referidas a los derechos humanos, del niño, de la mujer y DDSSRR. Además del entramado normativo entre la Ley de SSyPR y la Ley de Protección Integral de los Derechos de los niños y adolescentes que operan como antecedentes inmediatos, de tal modo que legitiman la necesidad de incluir la educación sexual en las escuelas y la concepción de los estudiantes como sujetos plenos de derecho, resulta significativa la referencia a la CEDAW dado que habilita el ingreso de la perspectiva de género. La ratificación de esta convención había sido un objeto de disputa entre los movimientos feministas y de mujeres y la jerarquía eclesiástica, que la interpretaba como la antesala de la legalización del aborto y la destrucción de la familia. Su incorporación en el texto de la Ley funciona como una condición que habilita el ingreso de la perspectiva de género a esta política pública, ya que legitima el abordaje educativo de las prácticas discriminatorias que tienen como objeto a la mujer.

Otro de los aspectos relevantes de esta ley es la relación que se establece entre la ESI y las familias. Teniendo en cuenta que uno de los temas instalados en el debate había sido la función del Estado y la familia con respecto a la educación sexual de los estudiantes, la normativa no solo circunscribe la ESI bajo la órbita estatal, sino que también posiciona la familia como destinataria de acciones de formación en la temática.

Artículo $9^{\circ}$. - (...) deberán organizar en todos los establecimientos educativos espacios de formación para los padres o responsables que tienen derecho a estar informados. Los objetivos de estos espacios son: (...) b) Promover la comprensión y el acompañamiento en la maduración afectiva del niño, niña y adolescente ayudándolo a formar su sexualidad y preparándolo para entablar relaciones interpersonales positivas; c) Vincular más estrechamente la escuela y la familia para el logro de los objetivos del programa. (Ley n. ${ }^{\circ} 26.150,2006$ )

Con respecto a los enunciados de este artículo, consideramos relevantes en primer lugar las designaciones del beneficiario: "los padres o responsables" y "la familia". La designación de familia en singular junto a padres en masculino plural

refiere al sintagma "ideología de género" que es definido como la promoción de la desnaturalización de las identidades sexuales llevada adelante por el feminismo y "las minorías sexuales" mediante la divulgación de doctrinas falsas. Por otro lado, el sintagma "igualdad de trato y oportunidades para varones y mujeres" goza de una aceptabilidad generalizada en el discurso social proveniente de relación con la formación discursiva de los derechos humanos. 
constituirían también uno de los objetos de disputa en las instancias subsiguientes de implementación de esta política, ya que se sostendría que tales designaciones remiten a una noción de familia nuclear heterosexual modélica instalada como pauta de normalidad, mientras que el plural "familias" ampliaría la referencia a diferentes configuraciones familiares. Por otra parte, resultan relevantes los enunciados acerca de los procesos madurativos y la formación de la sexualidad de los niños y adolescentes. En este sentido, los enunciados del objetivo "b" de este artículo plantean una concepción evolutiva de la sexualidad de estos sujetos, signada de manera específica por procesos madurativos y formativos, y articulada con el discurso de la psicología evolutiva. De manera coherente con este planteo, el inciso "b" del artículo 8 de esta ley menciona que cada jurisdicción implementará las propuestas de enseñanza en función de "las necesidades de los grupos etarios" (Ley n. ${ }^{\circ} 26.150,2006$ ). A lo largo del debate de esta normativa tuvo una presencia significativa la noción de adecuación por edad: si bien el texto de la Ley no le otorga a ello un lugar relevante, en los lineamientos curriculares y en los documentos posteriores la estratificación etaria cumpliría un rol estructurante.

Uno de los espacios más ajustados del debate precedente a la sanción de esta ley lo constituyó la definición de los contenidos que serían impartidos en las aulas. Tal como lo hemos constatado en este recorrido por los aspectos más relevantes de su breve articulado, más allá de algunas indicaciones generales que señalan la inclusión de contenidos referentes a la anatomofisiología humana, los procesos madurativos, la prevención de problemas de salud sexual y reproductiva y la igualdad entre varones y mujeres, la Ley no explicita de manera precisa y detallada los contenidos prescriptos para las diferentes etapas del sistema educativo, sino que delega esta tarea a otra instancia. En este sentido, el artículo $6 .^{\circ}$ establece que "los lineamientos curriculares básicos" de la ESI serán definidos por el Ministerio de Educación en consulta con el Consejo Federal de Cultura y Educación. Además, el artículo 7. plantea que la definición de esos lineamientos será asesorada por una "comisión interdisciplinaria de especialistas en la temática" convocada por el Ministerio de Educación. Con la anticipación de estas instancias de producción, las disputas centrales referidas a la definición de los contenidos de la ESI son desplazadas hacia un nuevo escenario destinado a la curricularización, enmarcado por las condiciones de restricción y de posibilidad de este aparato normativo. 


\section{Conclusiones}

La formulación de la ESI como una política pública ocurre en una arena de disputas que atraviesa diferentes contingencias, pero que refracta a su vez la profundidad de una memoria discursiva cuyos sentidos emergen y se actualizan. Con la reforma constitucional de 1994, el discurso jurídico argentino se entrama en una articulación de legitimidades y aceptabilidades transnacionales referidas a la sexualidad, cuyo principal efecto es la inscripción de los derechos sexuales y (no) reproductivos en el registro discursivo de los derechos humanos. Este movimiento reconfigura en un nuevo vocabulario de derechos el espacio discursivo de disputas históricas entre la Iglesia católica y los movimientos de mujeres en su interlocución con el Estado, con lo cual se da lugar a un juego táctico de operaciones acerca de la definición de la sexualidad integral, de los sujetos de su ejercicio y de los sujetos y mecanismos para su regulación.

En primer lugar, hemos descrito la conformación del espacio de enfrentamientos en torno a la Ley de SSyPR donde las formaciones discursivas de la biomedicina y los derechos humanos, en un juego de posiciones entre la Iglesia católica y los movimientos sociosexuales, traban relaciones de tensión y solapamiento en procesos de producción de sentido que, a su turno, trazan los contornos de lo legítimo y lo aceptable acerca de la intervención estatal en la sexualidad de la población. Los argumentos de esta disputa con sus sedimentos históricos y sus operaciones de actualización anticipan las zonas más constreñidas de un debate cuya intensidad quedaría condensada en el texto de la Ley de ESI. Así, la disputa por la gestión de la sexualidad que separa las aguas entre la Iglesia y la familia versus la injerencia estatal marca diferencias medulares sobre los sentidos que articulan sexualidad y reproducción, aunque también permite encontrar presupuestos compartidos acerca de la sexualidad y el riesgo.

La lectura propuesta aquí a una normativa que por primera vez vincula sexualidad y educación en una trama significante, regida por los derechos humanos, que nos permitió identificar los posicionamientos construidos por relaciones interdiscursivas y sus desplazamientos marcados por el avance de una agenda de progresiva ampliación de derechos. A su vez, este análisis permite visibilizar tensiones constitutivas del texto jurídico que condensan disputas pasadas, al tiempo que anticipa un horizonte de enfrentamientos acerca de los sentidos, los límites y los alcances de la ESI que se encuentran vigentes en nuestra sociedad. 


\section{Referencias}

Alma, Amanda; Lorenzo, Paula (2009). Mujeres que se encuentran: una recuperación histórica de los Encuentros Nacionales de Mujeres en Argentina (1986-2005). Buenos Aires: Feminaria Editora.

Angenot, Marc (1989). 1889: Un état du discours social. Montreal: Le Préambule.

Boccardi, Facundo (2010). La sexualidad en la red de los discursos mediáticos. Una lectura de la construcción de la educación sexual en la prensa argentina. Revista F@ro, 12, 1-7. Recuperado de http://web.upla.cl/revistafaro/n12/arto6.htm

Boccardi, Facundo; Boccardi, Paula (2011). Tensiones sexuales de la familia. Una lectura de las configuraciones de la familia en el discurso social argentino. Perspectivas de la Comunicación, 4(2), 68-78. Recuperado de http://revistas.ufro.cl/ojs/index.php/perspectivas/article/ view/119/1029

Boccardi, Facundo (2013). Educación de la diferencia sexual. Acerca de lo que se dice que hay que enseñar a la hora de dictar educación sexual en las escuelas. Revista Punto Género, 3, 83-97. https://www.doi.org/10.5354/0719-0417.2013.30268

Boccardi, Facundo (2020). Lo "sexual" y lo "reproductivo": Una genealogía de las definiciones de sexualidad en la arena discursiva internacional de los derechos. KAIROS. Revista de Temas Sociales, 24(46), 4-33. Recuperado de http://www.revistakairos.org/wp-content/ uploads/Lo-sexual-y-lo-reproductivo.pdf

Barros, Mercedes (2012). Human rights movement and discourse: its emergence and constitution in Argentina. Villa María: Eduvim.

Bellucci, Mabel (2014). Historia de una desobediencia. Abortoy feminismo. Buenos Aires: Capital Intelectual.

Brown, Josefina (2008). Los derechos (no) reproductivos en Argentina: encrucijadas teóricas y políticas. Cadernos Pagu, 30, 269-300.

Brown, Josefina (2014). Mujeres y ciudadanía en Argentina. Debates teóricos y políticos sobre derechos (no) reproductivos y sexuales (1990-2006). Buenos Aires: Teseo.

Butler, Judith (2002). Cuerpos que importan. Sobre los límites materiales y discursivos del "sexo". Buenos Aires: Paidós.

Cedes (2004). Resumen Ejecutivo 2004/4. Encuesta de opinión pública Saludy DDSSRR. Buenos Aires: Cedes.

Conferencia Episcopal Argentina (1985). Educacióny Proyecto de Vida, Tomo XIII. Buenos Aires: Conferencia Episcopal Argentina. 
Conferencia Episcopal Argentina (2006). El desafio de educar en el amor. Buenos Aires: Conferencia Episcopal Argentina.

Courtine, Jacques (1981). Quelques problèmes théoriques et méthodologiques en analyse du discours, à propos du discours communiste adressé aux chrétiens. Langages, 62, 9-128.

Decreto Reglamentario n. ${ }^{\circ} 1282$ (2003). Boletín Oficial $n .^{\circ} 30157 / 03$.

Dvoskin, Gabriel (2013). Paradigmas en disputa, presupuestos compartidos. ALED Revista Latinoamericana de Estudios del Discurso, 13(1), 79-98.

Esquivel, Juan (2004). Detrás de los muros. La Iglesia católica en tiempos de Alfonsín y Menem. Bernal: UNQ.

Esquivel, Juan; Alonso, Juan (2015). Actores y discursos religiosos en la esfera pública: los debates en torno a la educación sexual y a la 'muerte digna' en Argentina. Sexualidad, Salud y Sociedad (Rio de Janeiro), 21, 85-110.

Esquivel, Juan (2013). Cuestión de educación (sexual). Pujas y negociaciones político-religiosas en la Argentina democrática. Buenos Aires: Clacso.

Faur, Eleonor (2007). Fundamentos de derechos humanos para la educación integral de la sexualidad. En Educación sexual en la escuela. Perspectivas y reflexiones (pp. 23-46), dirigido por Ana Clement. Buenos Aires: Gobierno de la Ciudad de Buenos Aires.

Feldfeber, Myriam; Gluz, Nora (2011). Las políticas educativas en Argentina: herencias de los '9o, contradicciones y tendencias de "nuevo signo". Revista Educação \& Sociedade, 32 (115), 339-356.

Filippini, Leonardo (2011). Los abortos no punibles en la reforma constitucional de 1994. En Aborto y justicia reproductiva (pp. 399-418), compilado por Paola Bergallo. Buenos Aires: Del Puerto.

Foucault, Michel (2007). Historia de la sexualidad 1: la voluntad de saber. Buenos Aires: Siglo XXI.

Laudano, Claudia (1998). Cuando el aborto está en los medios... En Nuestros cuerpos, nuestras vidas: propuestas para la promoción de los derechos sexuales y reproductivos (pp. 119-130). Buenos Aires: Foro por los Derechos Reproductivos.

Ley Federal de Educación n. 24.195 (1993). Boletín Oficial n. ${ }^{\circ} 27632 / 93$.

Ley Nacional de Educación n. ${ }^{\circ} 26.206$ (2006). Boletín Oficial n. ${ }^{\circ}$ 31062/o6.

Ley Nacional de Educación Sexual Integral n. 26.150 (2006). Boletín Oficial n. ${ }^{\circ}$ 31017/o6.

Ley Nacional de la Convención sobre los derechos del niño n. 23.849 (1990). Boletín Oficial $n .^{\circ} 26993 / 90$.

Ley Nacional de los Derechos del Niño n. 25763 (2003). Boletín Oficial n. ${ }^{\circ}$ 30219/o3. 
Ley Nacional de Protección Integral de los Derechos de Niñas, Niños y Adolescentes n. ${ }^{\circ}$ 26.061 (2005). Boletín Oficial $.^{\circ} 30767 / 05$.

Ley Nacional de Salud Sexual y Procreación Responsable n. 25673 (2002). Boletín Oficial $n$. $^{\circ}$ 30032/02.

Ley Nacional de SIDA n. ${ }^{\circ} 23.798$ (1990). Boletín Oficial n. ${ }^{\circ} 26972 / 90$.

Ley Nacional sobre la Convención sobre eliminación de todas las formas de discriminación contra la mujer n. ${ }^{\circ} 23.179$ (1985). Boletín Oficial $n .^{\circ} 25690 / 85$.

Llobet, Valeria (2010). ¿Fábricas de niños? Las instituciones para menores en la era de los derechos. Buenos Aires: Novedades Educativas.

Mallimaci, Fortunato (2012). Política y catolicismo en el gobierno del Dr. Kirchner. En: Religión, política y cultura en América Latina (pp.169-197), editado por Cristian Parker. Santiago de Chile: USC-ACSRM.

Millet, Kate (1975). Política sexual. Ciudad de México: Aguilar.

Minyersky, Nelly (2003). Derecho de familia y aplicación de las Convenciones internacionales sobre niños y mujeres. En Derechos universales, realidades particulares. Reflexiones y herramientas para la concreción de los derechos humanos de niños, niñas y mujeres (pp. 98109), compilado por Eleonor Faur; Alicia Lamas y Gladys Acosta. Buenos Aires: Unicef.

Monte, María; Gavernet, Leticia (2015). Constituyentes y Constituidas: Sexualidad y Reproducción en las Reformas Constitucionales de Argentina (1994) y Bolivia (2009). Sortuz. Oñati Journal of Emergent Socio-legal Studies, 7(2), 42-55.

Organización Panamericana de la Salud - OPS; Organización Mundial de la Salud - OMS (2000). Promoción de la Salud Sexual: Recomendaciones para la acción. Antigua Guatemala, Guatemala: Organización Panamericana de la Salud - OPS.

Orlandi, Eni (2003). As formas do silencio. Campinas: Editora Unicamp.

Pecheny, Mario (2011). State and sexual politics in Latin America: the challenges of breaking through. Culture, Health and Sexuality, 13, 10-20.

Pecheny, Mario; Jones, Daniel; Ariza, Lucía (2016). Sexualidad, política y actores religiosos en la Argentina post-neoliberal (2003-2015). En Sexo, Delitos y Pecados: Intersecciones entre religión, género, sexualidad y el derecho en América Latina (pp.52-88), editado por Magdalena Sáez y José Morán Faúndes. Washington D.C.: Center for Latin American \& Latino Studies.

Peñas-Defagó, María; Campana, Maximiliano (2011). Programa Nacional de Salud Sexual y Procreación Responsable. Análisis de los debates en los medios de prensa de la sanción de la Ley 25.673. En Actoresy discursos conservadores en los debates sobre sexualidady reproducción en Argentina (pp. 23-47), coordinado por María Peñas-Defago y Juan Marco Vaggione. Córdoba: Ferreyra Editores. 
Petracci, Mónica (2004). Salud, derechos y opinión pública. Buenos Aires: Norma.

Petracci, Mónica; Pecheny, Mario (2007). Argentina, derechos humanos y sexualidad. Buenos Aires: Cedes.

Puga, Mariela; Vaggione, Juan (2013). La política de la conciencia. La objeción como estrategia contra los derechos sexuales y reproductivos. En Peripecias en la lucha por el derecho al aborto (pp. 93-138), editado por Marta Vassallo. Colección Religión Género y Sexualidad. Córdoba: Editorial Ferreyra.

Sesión Interruptus (2005, 10 de noviembre). Página 12, p. 14.

Si es sexual, nada de educación. (6 de octubre de 2006). Página 12, p. 18.

Tedesco, Juan (Comp.) (2015). La educación argentina hoy. La urgencia del largo plazo. Buenos Aires: Siglo XXI.

Terigi, Flavia (2016). Políticas públicas en Educación tras doce años de gobierno de Néstor Kirchner y Cristina Fernández. Buenos Aires: Fundación Friedrich Ebert.

Vaggione, Juan (2005). Reactive Politicization and Religious Dissidence. The Political Mutations of the Religious. Social Theory and Practice, 32(2), 233-255.

Wainerman, Catalina; Di Virgilio, María; Chami, Natalia (2008). La escuela y la educación sexual. Buenos Aires: Manantial. 